\section{Analysis of the Effect of Locking Compression Plate Internal Fixation for the Treatment of Limb Fractures}

Sir,

Limb fracture is a common condition, mostly caused by violent injury. Traditional open reduction and internal fixation uses mostly periosteal peeling and direct reduction to ensure the stability of the fracture end. However, the operation can cause incision infection with poor fracture healing and other complications, which is not conducive to the improvement of prognosis. Locking compression plate is an open reduction and internal fixation method, developed on the basis of dynamic compression plate and point contact plate. ${ }^{1}$ The compression locking plate is designed to achieve elastic fixation at the fracture end, which not only can effectively stabilize the fracture end, but also stimulate bone regeneration and promote fracture healing. Some scholars have reported the clinical effect of compression locking plate internal fixation. ${ }^{2}$ However, the understanding of the concept of compression locking plate is still incomplete and there is a lack of long-term clinical follow-up. The author followed up the patients with limb fractures after internal fixation with locking compression plate for an average of 24 months, and observed that the effect of this method was satisfactory.

This descriptive and analytical study was carried out at the Department of Orthopedics, Tianjin Fifth Central Hospital, China, from February 2015 to May 2016, approved by the Hospital Ethics Committee. A total of 97 patients with limb fractures were selected as the study subjects. The inclusion criteria were that patients were diagnosed as limb fractures by clinical examination and no operative contraindications. Patients with renal, hepatic and cardiac dysfunction; those who cannot tolerate surgery completely; lactating or pregnant women; those who cannot complete follow-up after surgery were excluded.

All the patients were treated with locking compression plate internal fixation; that is, the patients were placed on the traction bed, closed traction reduction was firstly done and the force line and length of the affected limbs were maintained. A small incision was made at the proximal or distal end of the fracture and separated layer by layer; a locking compression plate was inserted along the submuscular and periosteal spaces. During the operation, the position of the plate was confirmed by fluoroscopy C-arm X-ray machine, and 2-4 monocortical locking screws were placed far away from the fracture site. Bicortical locking screw could be considered for elderly patients with osteoporosis. The fracture reduction should be confirmed to be good, the incision was sutured layer by layer, and routine anti-infection treatment was given after operation.

Among the 97 patients, 55 were males (56.70\%) and 42 were females $(43.30 \%)$. The age was ranged from 21 to 65 with an average age of $47.84 \pm 3.62$ years. Fracture types: 41 patients $(42.27 \%)$ were proximal humerus fracture, 31 patients $(31.96 \%)$ were forearm ulna and radius fracture, and 25 patients $(25.77 \%)$ were tibia and fibula fracture. According to causes of injury, 48 cases $(49.48 \%)$ were traffic accident injuries, 30 cases $(30.93 \%)$ were fall injuries, 17 cases $(17.53 \%)$ were sport injuries and 2 cases (2.06\%) were other injuries.

All the patients were followed up for an average of 24 months (21-30 months); none was lost to follow-up. X-ray examination showed callus formation in $87(89.69 \%)$ of the 97 patients about three weeks after operation and a large callus formed at about nine weeks. All patients achieved bone healing without complications such as nail breaking and loosening of internal fixation. Only one patient $(1.03 \%)$ with lower tibial fracture had incision infection after operation and healed after intensive dressing change. None of the patients received bone graft.

A study showed that compared with the locking compression plate, the failure of internal fixation of traditional steel plate occurred earlier. ${ }^{3}$ This study found that locking compression plate was an ideal internal fixation method for the treatment of limb fractures. This conclusion is basically consistent with the research report of Kim et al. 4

The author's clinical experience is that the locking compression plate does not contact the bone surface and need precise shaping; therefore, it will not compress the periosteum and affect the blood supply at the fracture end. ${ }^{5}$ In addition, by eccentric fixation of compression holes and common standard screws, the fragments can be compressed with each other. Combined application of compression holes and locking holes not only can achieve close alignment between fragments, but also form a stable whole between the fragments and the whole plate. In the process of internal fixation of locking compression plate, as the screw and plate are locked as a whole, the stress transmission is transmitted through the proximal screw through the plate in the fracture area to the distal screw, thus protecting the microenvironment of fracture healing in the fracture area, which is conducive to fracture healing. 


\section{REFERENCES}

1. Zderic I, Oh JK, Stoffel K, Sommer C, Helfen T, Camino G, et al. Biomechanical analysis of the proximal femoral locking compression plate: Do quality of reduction and screw orientation influence construct stability? J Orthop Trauma 2018; 32:67-74.

2. Naseer MK, Sah RK, Nadeem RD, Awais SM, Akhtar M. Effectiveness of locking compression plate in wedge fracture of diaphysis of radius and ulna in adults: A descriptive case series. J Pak Med Assoc 2017; 67:1767-70.

3. Gardner MJ, Brophy RH, Campbell D, Mahajan A, Wright TM, Helfet DL, et al. The mechanical behavior of locking compression plates compared with dynamic compression plates in a cadaver radius model. J Orthop Trauma 2005; 19:597-603.

4. Kim HJ, Oh JK, Hwang JH, Park YH. The use of T-LCP (locking compression plate) for the treatment of the lateral malleolar fractures. Eur J Orthop Surg Traumatol 2013; 23:233-7.
5. Hasan O, Fahad S, Umer M, Rashid H. Modified use of proximal humeral interlocking plate (PHILOS) in lateral malleolus fracture, functional and radiological outcome in series of 13 cases. Int J Surg 2018; 56:79-82.

Li Tan' ${ }^{1}$ Kai Yul, Jie Yang', Jian Yangl and Dianying Zhang ${ }^{2}$

1 Department of Orthopedics, Tianjin Fifth Central Hospital, Tianjin, 300450, China

2 Department of Orthopedics and Trauma, People's Hospital, Peking University, Beijing 100044, China

Correspondence: Dianying Zhang, Department of Orthopedics and Trauma, People's Hospital, Peking University, Beijing 100044, China

E-mail:jkker1@163.com

Received: December 14, 2018; Revised: January 28, 2019; Accepted: February 08, 2019 